CTP-TAMU-16/99, SINP-TNP/99-14

hep-th/9905014

\title{
((F, D1), D3) Bound State and Its T-dual Daughters
}

\author{
J. X. Lu ${ }^{1}$ and Shibaji Roy ${ }^{2}$ \\ ${ }^{1}$ Center for Theoretical Physics, Texas AEM University, College Station, TX 77843 \\ E-mail: jxlu@rainbow.physics.tamu.edu \\ ${ }^{2}$ Saha Institute of Nuclear Physics, 1/AF Bidhannagar, Calcutta 700 064, India \\ E-mail: roy@tnp.saha.ernet.in
}

\begin{abstract}
In the previous paper hep-th/9904129 , we constructed a general explicit BPS solution for (F, D3) non-threshold bound state. By using the $\mathrm{SL}(2, \mathrm{Z})$ symmetry of type IIB string theory, we here construct from (F, D3) a more general BPS configuration for a D3 brane with certain units of quantized 5-form flux and an infinite number of parallel (F, D1)-strings. We study its decoupling limit and find that given Maldacena's $A d S_{5} / C F T_{4}$ correspondence with respect to simple D3 branes and with the usual string coupling, we should have a similar correspondence with respect to this bound state but now with an effective string coupling. We discuss possible descendants of this bound state by T-dualities along its longitudinal or transverse directions. In particular, we present explicit configurations for $((\mathrm{F}, \mathrm{Dp}), \mathrm{D}(\mathrm{p}+2))$ bound states for $2 \leq p \leq 5$. All these configurations preserve one half of the spacetime supersymmetries.
\end{abstract}




\section{INTRODUCTION}

In the previous paper [1], we showed that for the non-threshold bound state (F, D3) the dilaton in general does not remain constant as opposed to a simple D3 brane configuration, even though in both cases one half of the spacetime supersymmetries are preserved. However, the dilaton was still found to be bounded from below by its asymptotic value and from above by its finite horizon value. The effective string coupling, defined as $g_{\text {eff }}=e^{\phi}$, decreases its value when we move from asymptotic region to the horizon region. This is caused by the infinite number of F-strings in the bound state. In the so-called decoupling limit, this bound state is expected to be described either by fields in the bulk spacetime or by the $\mathcal{N}=4 U(n)$ SYM theory in $1+3$ dimensions (with $n$ the 5 -form charge associated with the 3 -brane in (F, D3)). In the bulk, we need to study only the near-horizon region under this limit. We found that the near-horizon string metric of (F, D3) is not automatically $A d S_{5} \times S^{5}$ but under some constant rescalings of the coordinates it becomes $A d S_{5} \times S^{5}$. The resulting metric then becomes identical with the near-horizon geometry of an equivalent simple D3 brane with 5-form charge $n$ with the effective string coupling evaluated at the horizon rather than the usual string coupling. Following the symmetry argument of Maldacena [2], we mentioned that we must have an $A d S_{5} / C F T_{4}$ correspondence with respect to this 'effective' vacuum configuration of the equivalent simple D3 brane. The gauge coupling for the SYM theory becomes related to the effective string coupling rather than the usual string coupling as $g_{\mathrm{YM}}^{2}=2 \pi g_{\mathrm{eff}}$.

After the rescalings of the coordinates, the information about the F-strings in the (F, D3) in the metric disappeared completely. The only difference between the equivalent simple D3 brane with the usual simple D3 brane just mentioned is the string coupling constant. So the effect of the F-strings must be encoded into this effective string coupling. Indeed, we found that the tension for this equivalent simple D3 brane is nothing but the tension for the (F, D3) bound state. This indicates that the 'effective' vacuum mentioned above is just the (F, D3) bound state. The vacuum configuration for the SYM theory in that case does 
not contain the electric flux lines. We also found that the effect of the electric flux lines in the worldvolume picture of the (F, D3) bound state gets absorbed into the gauge coupling constant $g_{\mathrm{YM}}^{2}=2 \pi g_{\mathrm{eff}}$. Therefore, we had a consistent picture both in the bulk and on the brane.

Thus the $A d S_{5} / C F T_{4}$ correspondence of Maldacena was found to hold true even for this non-trivial D3 brane configuration, namely, (F, D3) but with the corresponding effective string coupling rather than the usual string coupling. We also observed that the effective string coupling is quantized in terms of the integral charges associated with the F-strings and the D3 brane in the (F, D3) bound state. In particular, it can be independent of the usual string coupling in certain limit.

In testing Maldacena's $A d S_{5} / C F T_{4}$ conjecture, most of the current activities are focused on relating the perturbative particle-like modes of type IIB supergravity on $A d S_{5} \times S^{5}$ with the operators of the boundary $\mathcal{N}=4 \mathrm{SYM}$ theory. In [1], we took a different step in relating non-trivial (non-perturbative) (F, D3) configuration in the bulk to the corresponding one in SYM on the boundary. Actually, there already exist some efforts [4] in this direction, for example, in [6.7] certain effects of D-instantons of type IIB theory in the decoupling limit have been checked to match with those of the instantons in YM theory on the boundary.

In this paper, we continue our study for a more general D3 brane configuration, namely, the non-threshold ((F, D1), D3) bound state. We construct this configuration explicitly in the following section by using the type IIB SL(2,Z) symmetry and the (F, D3) configuration given in [1]. In section 3, we study the properties of this ((F, D1), D3) bound state. In section 4 , we study the decoupling limit of this bound state. We find that the $A d S_{5} / C F T_{4}$ correspondence holds true in the same spirit as in [2] for this ((F, D1), D3) bound states but once again with the corresponding effective string coupling rather than the usual string coupling. In particular, we perform a non-trivial check of this correspondence by showing that the gauge coupling and theta angle obtained from the corresponding $\mathcal{N}=4 \mathrm{SYM}$ are indeed related to the effective string coupling and value of the RR scalar evaluated at the horizon, as required by the correspondence. The effective string coupling is also quantized 
as in [1] in terms of integral charges associated with the F-strings, D-strings and D3 brane in the ((F, D1), D3) bound state. Interestingly, we find that D-strings in the ((F, D1), D3) tend to increase the effective string coupling while the F-strings tend to decrease it. This is also true for the value of the RR scalar evaluated at the horizon. In section 5 , we present all possible descendants of this ((F, D1), D3) bound state by T-dualities. We also discuss some BPS states which can be obtained by the type IIB SL(2,Z) acting on the descendants of the $((\mathrm{F}, \mathrm{D} 1), \mathrm{D} 3)$ in the non-perturbative type IIB string theory. In particular, we find non-threshold (Dp, Dp) bound states for $1 \leq p \leq 8$, where the two Dp's share $(p-1)$ common directions. We also find non-threshold (Dp, Dp, Dp) bound states for $1 \leq p \leq 7$, where the three Dp's share $(p-1)$ directions; non-threshold (Dp, Dp, Dp, Dp) bound states for $1 \leq p \leq 6$, where the four Dp's share also $(p-1)$ common directions and so on. This can be continued until we reach a seemingly unique non-threshold (D1, D1, D1, D1, D1, D1, D1, D1, D1) bound state with the nine D1's sharing no common direction. All these bound states preserve one half of the spacetime supersymmetries. We discuss the possible role of these bound states in the M- or U-theory. We also present the explicit configurations for the $((\mathrm{F}, \mathrm{Dp}), \mathrm{D}(\mathrm{p}+2))$ bound states for $2 \leq p \leq 5$. Finally, we conclude this paper in section 6 .

\section{NON-THRESHOLD ((F, D1), D3) BOUND STATE}

In this section, we will construct the non-threshold ((F, D1), D3) bound state using the type IIB SL(2,Z) symmetry and the (F, D3) solution given in [1]. We will follow the procedure outlined in [8,9]. We take the initial configuration as (F, D3) solution, with vanishing asymptotic values for all non-vanishing fields. This is given by the following Einstein metric,

$$
d s^{2}=\left(H^{\prime} H\right)^{1 / 4}\left[H^{-1}\left(-\left(d x^{0}\right)^{2}+\left(d x^{1}\right)^{2}\right)+H^{\prime-1}\left(\left(d x^{2}\right)^{2}+\left(d x^{3}\right)^{2}\right)+d y^{i} d y^{i}\right]
$$

with $i=1, \cdots, 6$; the dilaton, 


$$
e^{\phi}=\left(\frac{H^{\prime}}{H}\right)^{1 / 2}
$$

and the remaining non-vanishing fields,

$$
\begin{aligned}
H_{3}^{(1)} & =-m \Delta_{(m, n)}^{-1 / 2} d H^{-1} \wedge d x^{0} \wedge d x^{1}, \\
H_{3}^{(2)} & =m n \Delta_{(m, n)}^{-1} H^{\prime-2} d H \wedge d x^{2} \wedge d x^{3}, \\
H_{5} & =n \frac{\sqrt{2} \kappa_{0} Q_{0}^{3}}{\Omega_{5}}\left(* \epsilon_{5}+\epsilon_{5}\right) .
\end{aligned}
$$

In the above, $H_{3}^{(1)}$ and $H_{3}^{(2)}$ are the NSNS and RR 3-form field strengths, respectively, and they form a doublet

$$
\mathcal{H}_{3}=\left(\begin{array}{c}
H_{3}^{(1)} \\
H_{3}^{(2)}
\end{array}\right),
$$

under the classical SL(2,R) symmetry of type IIB string theory. $H_{5}$ is the self-dual 5 -form field strength which is inert under either $\mathrm{SL}(2, \mathrm{R})$ or $\mathrm{SL}(2, \mathrm{Z}) . H$ is a Harmonic function

$$
H=1+\frac{Q_{3}}{r^{4}}
$$

with $r^{2} \equiv y^{i} y^{i}$ and $Q_{3}=\Delta_{(m, n)}^{1 / 2} \sqrt{2} \kappa_{0} Q_{0}^{3} /\left(4 \Omega_{5}\right), H^{\prime}$ is a second Harmonic function defined as,

$$
H^{\prime}=1+\frac{n^{2} Q_{3} / \Delta_{(m, n)}}{r^{4}}
$$

Also in the above, the $\Delta$-factor has the form,

$$
\Delta_{(m, n)}=m^{2}+n^{2}
$$

where the asymptotic values of the scalars have been set to zero. Here $n$ is the quantized 5-form flux or 3-brane charge and $m$ is the quantized NS string charge or the number of F-strings through a $(2 \pi)^{2} \alpha^{\prime}$ area of the 2-dimensional plane perpendicular to the strings in (F, D3) as discussed in [1]. $m$ and $n$ are relatively prime integers. Unless stated otherwise, * always denotes the Hodge dual. $\epsilon_{n}$ denotes the volume form on an $n$-sphere and the volume of a unit $n$-sphere is 


$$
\Omega_{n}=\frac{2 \pi^{(n+1) / 2}}{\Gamma((n+1) / 2)} .
$$

In the above $\sqrt{2} \kappa_{0}=(2 \pi)^{7 / 2} \alpha^{\prime 2}$ and $Q_{0}^{p}$ is the unit charge for a Dp-brane defined as,

$$
Q_{0}^{p} \equiv(2 \pi)^{(7-2 p) / 2} \alpha^{\prime(3-p) / 2}
$$

It is well-known that type IIB supergravity possesses a classical $\mathrm{SL}(2, \mathrm{R})$ symmetry 10 and a discrete subgroup $\mathrm{SL}(2, \mathrm{Z})$ of this group is now believed [11] to survive as a quantum symmetry of the non-perturbative type IIB string theory. Under a global SL(2,R) transformation $\Lambda$, we have the following transformations for the Einstein metric $g_{\mu \nu}$, the 3-form field strength doublet $\mathcal{H}$, the 5 -form field strength $H_{5}$ and the scalar matrix $\mathcal{M}$ parameterizing the coset $\mathrm{SL}(2, \mathrm{R}) / \mathrm{SO}(2)$ as

$$
g_{\mu \nu} \rightarrow g_{\mu \nu}, \quad \mathcal{M} \rightarrow \Lambda \mathcal{M} \Lambda^{T}, \quad \mathcal{H} \rightarrow\left(\Lambda^{-1}\right)^{T} \mathcal{H}, \quad H_{5} \rightarrow H_{5}
$$

where the $\mathrm{SL}(2, \mathrm{R}) / \mathrm{SO}(2)$ coset scalar matrix is defined as,

$$
\mathcal{M}=e^{\phi}\left(\begin{array}{cc}
e^{-2 \phi}+\chi^{2} & \chi \\
\chi & 1
\end{array}\right),
$$

with $\phi$, the dilaton and $\chi$, the axion (or RR scalar) in type IIB supergravity.

Since there are an infinite number of (F, D1)-strings in ((F, D1), D3), as discussed in [1], the corresponding charges for F-strings and D-strings should be calculated according to

$$
e^{(i)}=\frac{1}{\sqrt{2} \kappa_{0}} \int_{R^{2} \times S^{5}}\left(\mathcal{M}^{i j} * H_{3}^{(j)}-\epsilon^{i j} B_{2}^{(j)} \wedge H_{5}\right)
$$

where $i, j=1,2$ and $\epsilon^{i j}$ is the totally antisymmetric $\mathrm{SL}(2, \mathrm{R})$ invariant tensor with $\epsilon^{12}=1$. In general, $e^{(i)}$ are infinite because of the infinite number of F-strings and D-strings in ((F, D1), D3). As discussed in [1], the following quantities $Q^{(i)}$ are nevertheless finite and quantized,

$$
Q^{(i)} \equiv(2 \pi)^{2} \alpha^{\prime} \frac{e^{(i)}}{\sqrt{2} \kappa_{0} A_{2}},
$$

where $A_{2}=\int d x^{2} d x^{3}$ is the coordinate area of the $x^{2} x^{3}$-plane. The quantities $Q^{(1)}$ and $Q^{(2)}$ represent charges associated with the F-strings and D-strings or the number of F-strings and 
D-strings through a $(2 \pi)^{2} \alpha^{\prime}$ area over the $x^{2} x^{3}$-plane measured in some units. From Eqs. (2.12) and (2.10), we find that the charge doublet $\mathcal{Q}^{T}=\left(Q^{(1)}, Q^{(2)}\right)$ will transform under an $\mathrm{SL}(2, \mathrm{R})$ transformation as

$$
\mathcal{Q} \rightarrow \Lambda \mathcal{Q}
$$

We will use the above transformations Eqs. (2.10) and (2.14) to obtain the non-threshold ((F, D1), D3) configuration from the given initial (F, D3) solution Eqs.(2.1)-(2.7). Since the 5 -form field strength is inert under the $\mathrm{SL}(2, \mathrm{R})$ transformation, we expect that the expression for $H_{5}$ given in Eq. (2.3) remains unchanged. In other words, the integer $n$ remains the same. However, the integral NS-string charge $m$ must be changed under the $\mathrm{SL}(2, \mathrm{R})$ transformation $\Lambda$. In general, it cannot remain as an integer, i.e, quantized. For this reason, one either needs to introduce a compensating factor for $m$ by hand [8] or take the initial configuration with an arbitrary classical charge $\tilde{\Delta}_{(p, q)}^{1 / 2}[9]$ in place of $m$ such that the resulting NS-string and D-string charges can be quantized. The process of charge quantization also determines this factor in terms of the quantized NS-string and RR-string charges and the asymptotic values of the dilaton and the axion. We will choose the latter approach, i.e., we take the integer $m$ as an arbitrary classical charge $\tilde{\Delta}_{(p, q)}^{1 / 2}$ in the above (F, D3) solution.

As usual, we start with the zero asymptotic values of $\phi$ and $\chi$, i.e., $\mathcal{M}_{0}=I$, with $I$ the unit matrix. Here $\mathcal{M}_{0}$ denotes the scalar matrix $\mathcal{M}$ when the scalars take their asymptotic values. We now seek a $2 \times 2 \operatorname{SL}(2, \mathrm{R})$ matrix $\Lambda_{0}$ which maps the zero asymptotic values of $\phi$ and $\chi$ to $\phi_{0}$ and $\chi_{0}$ (which are arbitrary but given) as

$$
\Lambda_{0} I \Lambda_{0}^{T}=\Lambda_{0} \Lambda_{0}^{T}=\mathcal{M}_{0}=e^{\phi_{0}}\left(\begin{array}{cc}
e^{-2 \phi_{0}}+\chi_{0}^{2} & \chi_{0} \\
\chi_{0} & 1
\end{array}\right) .
$$

The above equation fixes the $\mathrm{SL}(2, \mathrm{R})$ matrix $\Lambda_{0}$ in terms of $\phi_{0}, \chi_{0}$ and an undetermined $\mathrm{SO}(2)$ angle $\alpha$ as

$$
\Lambda_{0}=e^{\phi_{0} / 2}\left(\begin{array}{cc}
e^{-\phi_{0}} \cos \alpha+\chi_{0} \sin \alpha & -e^{-\phi_{0}} \sin \alpha \\
\sin \alpha & \cos \alpha
\end{array}\right) .
$$


Imposing charge quantization on $Q^{(i)}$ as $Q^{(1)}=p, Q^{(2)}=q$ with $p, q$ relatively prime integers for our general ((F, D1), D3) bound state, we have from Eq. (2.14)

$$
\left(\begin{array}{c}
p \\
q
\end{array}\right)=\Lambda_{0}\left(\begin{array}{c}
\tilde{\Delta}_{(p, q)}^{1 / 2} \\
0
\end{array}\right) .
$$

The above equation fixes not only the $\mathrm{SL}(2, \mathrm{R})$ matrix $\Lambda_{0}$ completely but also the $\Delta_{(p, q)}$-factor in terms of $p, q$ and $\phi_{0}, \chi_{0}$ as

$$
\Lambda_{0}=\frac{1}{\tilde{\Delta}_{(p, q)}^{1 / 2}}\left(\begin{array}{cc}
p & -q e^{-\phi_{0}}+\chi_{0}\left(p-\chi_{0} q\right) e^{\phi_{0}} \\
q & \left(p-\chi_{0} q\right) e^{\phi_{0}}
\end{array}\right)
$$

and

$$
\tilde{\Delta}_{(p, q)}=e^{\phi_{0}}\left(p-\chi_{0} q\right)^{2}+e^{-\phi_{0}} q^{2}
$$

which is invariant under an $\mathrm{SL}(2, \mathrm{Z})$ transformation. Note that in this process the $\mathrm{SO}(2)$ angle $\alpha$ gets fixed as $e^{i \alpha}=\left[\left(p-\chi_{0} q\right) e^{\phi_{0} / 2}+i q e^{-\phi_{0} / 2}\right] \tilde{\Delta}_{(p, q)}^{-1 / 2}$.

Once we have the $\mathrm{SL}(2, \mathrm{R})$ matrix $\Lambda_{0}$ (Eq. (2.18) ), we can obtain a general ((F, D1), D3) configuration by performing the $\mathrm{SL}(2, \mathrm{R})$ transformation as given in Eq. (2.10) with $\Lambda=\Lambda_{0}$ on the initial (F, D3) configuration given in Eqs. $(2.1)-(2.7)$, with $m$ replaced by $\tilde{\Delta}_{(p, q)}^{1 / 2}$. This configuration is characterized in terms of the quantized charges $p$ and $q$ associated with the F-strings and D-strings, the quantized charge $n$ of the D3 brane, and arbitrary but given asymptotic values $\phi_{0}$ and $\chi_{0}$ of the dilaton $\phi$ and the axion $\chi$, respectively. Thus, we have the following explicit configuration of the bound state ((F, D1), D3). The 5 -form $H_{5}$ remains unchanged, as given in Eq. (2.3). The Einstein metric $d s^{2}$ and the Harmonic functions $H$ and $H^{\prime}$ all remain the same in forms as before, but the $\Delta_{(m, n)}$-factor given in Eq. (2.7), which appears in the Harmonic functions, is changed to

$$
\begin{aligned}
\Delta_{(p, q, n)} & =\tilde{\Delta}_{(p, q)}+n^{2}, \\
& =\left(p-\chi_{0} q\right)^{2} e^{\phi_{0}}+q^{2} e^{-\phi_{0}}+n^{2},
\end{aligned}
$$

which is also invariant under $\mathrm{SL}(2, \mathrm{Z})$ as expected since $n$ is inert under $\mathrm{SL}(2, \mathrm{Z})$. The dilaton is now 


$$
e^{\phi}=e^{\phi_{0}} \frac{H^{\prime \prime}}{\sqrt{H H^{\prime}}},
$$

and the axion is

$$
\chi=\frac{\chi_{0} H^{\prime}+\left(H-H^{\prime}\right) p q e^{-\phi_{0}} / \tilde{\Delta}_{(p, q)}}{H^{\prime \prime}} .
$$

In the above, we have introduced a third Harmonic function $H^{\prime \prime}$ as

$$
H^{\prime \prime}=1+\frac{\left(n^{2}+q^{2} e^{-\phi_{0}}\right) Q_{3} / \Delta_{(p, q, n)}}{r^{4}}
$$

where $Q_{3}=\Delta_{(p, q, n)}^{1 / 2} \sqrt{2} \kappa_{0} Q_{0}^{3} /\left(4 \Omega_{5}\right)$. As expected, both the dilaton and the axion approach their respective asymptotic values as $r \rightarrow \infty$. Finally, the NSNS and RR 3-form field strengths are

$$
\begin{gathered}
H_{3}^{(1)}=-e^{\phi_{0}}\left(p-\chi_{0} q\right) \Delta_{(p, q, n)}^{-1 / 2} d H^{-1} \wedge d x^{0} \wedge d x^{1}-q n \Delta_{(p, q, n)}^{-1} H^{\prime-2} d H \wedge d x^{2} \wedge d x^{3} \\
H_{3}^{(2)}=\left[\chi_{0}\left(p-\chi_{0} q\right) e^{\phi_{0}}-q e^{-\phi_{0}}\right] \Delta_{(p, q, n)}^{-1 / 2} d H^{-1} \wedge d x^{0} \wedge d x^{1} \\
\quad+p n \Delta_{(p, q, n)}^{-1} H^{\prime-2} d H \wedge d x^{2} \wedge d x^{3} .
\end{gathered}
$$

In the following section, we will study certain properties of this configuration.

\section{PROPERTIES OF ((F, D1), D3) BOUND STATE}

By construction, we know that the configuration of ((F, D1), D3) given in the previous section carries one $(p, q)$-string along the $x^{1}$-axis per $(2 \pi)^{2} \alpha^{\prime}$ area over the $x^{2} x^{3}$-plane. There are $p$ F-strings and $q$ D-strings in the $(p, q)$-string. This non-threshold bound state also carries the 5 -form charge $n$.

We would like to mention that in constructing the $\mathrm{SL}(2, \mathrm{Z})$ invariant ((F, D1), D3) bound state solution of type IIB theory, we have chosen the Einstein-frame metric to be asymptotically Minskowski. However, since we are here studying strings and Dp branes in string theory, it would be natural to choose the string-frame metric to be asymptotically Minkowski instead. Moreover, we need to calculate the tension for this bound state and 
compare it with the corresponding worldvolume result where the string-frame metric is always chosen to be asymptotically Minkowski in the context of string theory. Also, usually in discussing the so-called decoupling limit, the string-frame metric is chosen to be asymptotically Minkowski. For these reasons, we re-express the explicit solution given in the previous section in terms of such a choice for the asymptotic metrid" For this choice the 5-form field strength continues to be given by the expression in Eq. (2.3). The dilaton and the axion also remain the same as given by Eqs. (2.21) and (2.22), respectively. The same is true for the Harmonic functions $H, H^{\prime}$ and $H^{\prime \prime}$ but with the replacements $Q_{3} \rightarrow e^{3 \phi_{0} / 2} Q_{3}$, $n^{2} Q_{3} / \Delta_{(p, q, n)} \rightarrow n^{2} e^{\phi_{0} / 2} Q_{3} / \Delta_{(p, q, n)}$, and $\left(n^{2}+q^{2} e^{-\phi_{0}}\right) Q_{3} / \Delta_{(p, q, n)} \rightarrow\left(n^{2}+q^{2}\right) e^{\phi_{0} / 2} Q_{3} / \Delta_{(p, q, n)}$ in the respective Harmonic functions. Here the $\Delta_{(p, q, n)}$ is also changed to

$$
\Delta_{(p, q, n)}=\left(p-\chi_{0} q\right)^{2} e^{\phi_{0}}+\left(q^{2}+n^{2}\right) e^{-\phi_{0}}
$$

which is not manifestly $\mathrm{SL}(2, \mathrm{Z})$ invariant now. Apart from an overall constant factor $e^{-\phi_{0} / 2}$ and the changes in the corresponding Harmonic functions $H$ and $H^{\prime}$ mentioned above, the Einstein-frame metric Eq.(2.1) remains the same in form. The NSNS and RR 3-form field strengths are now changed to

$$
\begin{gathered}
H_{3}^{(1)}=-e^{\phi_{0} / 2}\left(p-\chi_{0} q\right) \Delta_{(p, q, n)}^{-1 / 2} d H^{-1} \wedge d x^{0} \wedge d x^{1}-e^{-\phi_{0}} q n \Delta_{(p, q, n)}^{-1} H^{\prime-2} d H \wedge d x^{2} \wedge d x^{3}, \\
H_{3}^{(2)}=e^{-\phi_{0} / 2}\left[\chi_{0}\left(p-\chi_{0} q\right) e^{\phi_{0}}-q e^{-\phi_{0}}\right] \Delta_{(p, q, n)}^{-1 / 2} d H^{-1} \wedge d x^{0} \wedge d x^{1} \\
+e^{-\phi_{0}} p n \Delta_{(p, q, n)}^{-1} H^{\prime-2} d H \wedge d x^{2} \wedge d x^{3}
\end{gathered}
$$

where $\Delta_{(p, q, n)}$ is given by Eq. (3.1).

We can now calculate the mass per unit 3-brane volume for this bound state with the new Einstein-frame metric using the generalized formula given in [1]. We therefore find the tension for the ((F, D1), D3) bound state as

\footnotetext{
${ }^{1}$ Actually there are some rationality behind the choice of the string-frame metric to be asymptotically Minkowski and relating such a choice to the $\mathrm{SL}(2, \mathrm{Z})$ symmetry of type IIB theory. We will give a detail account of this along with other things in a separate paper.
} 


$$
T_{3}(p, q, n)=\frac{T_{0}^{3}}{g} \sqrt{n^{2}+q^{2}+g^{2}\left(p-\chi_{0} q\right)^{2}}
$$

which, for $\chi_{0}=0$, agrees precisely with what we obtained from the worldvolume study in [3.

The expression for tension in (3.3) clearly shows that the spacetime ((F, D1), D3) bound state should be identified with the bound state consisting of D3 branes carrying quantized constant electric and magnetic fields given in [3] based on the worldvolume study. In other words, the $(p, q)$-strings should be identified with the quantized electric and magnetic flux lines. The quantized constant electric and magnetic fields are related to each other by the $\mathrm{SL}(2, \mathrm{Z})$ symmetry in the $\mathcal{N}=4 \mathrm{SYM}$ theory on the worldvolume. This is directly linked to the type IIB SL $(2, \mathrm{Z})$ relating F-strings and D-strings in the $(p, q)$-string provided we identify the gauge coupling and the $\theta$-angle in the gauge theory with the string coupling and the asymptotic value of the axion as

$$
\frac{2 \pi i}{g_{\mathrm{YM}}^{2}}+\frac{\theta}{4 \pi}=\frac{i}{g}+\chi_{0},
$$

where $g=e^{\phi_{0}}$ is the string coupling?]

\section{THE DECOUPLING LIMIT OF ((F, D1), D3) BOUND STATE}

Let us now study the decoupling limit for this ((F, D1), D3) bound state. Under this limit,

$$
g_{\mathrm{YM}}^{2}=2 \pi g=\text { fixed }, \quad U=\frac{r}{\alpha^{\prime}}=\text { fixed }, \quad \alpha^{\prime} \rightarrow 0,
$$

we know that the modes propagating on the 3-brane worldvolume decouple from the modes propagating in the bulk spacetime. Also since $\alpha^{\prime} \rightarrow 0$ is a low-energy limit, the modes

\footnotetext{
${ }^{2}$ Our convention for the complex gauge coupling follows from the Born-Infeld action which differs from the standard one for $S U(n)$ theories by a factor 2. We thank Juan Maldacena for discussion on this issue.
} 
propagating on the brane are just the massless ones of the open strings. Therefore, D3 brane is described by the $\mathcal{N}=4 \mathrm{SYM}$ theory. It is also described by the fields in the bulk. So we have two equivalent descriptions for the D3 brane.

From the spacetime point of view, the decoupling limit tells us that we need to study only the near-horizon geometry. Under this limit, the dilaton, which defines the effective string coupling $g_{\text {eff }}$, is given as,

$$
g_{\text {eff }} \equiv e^{\phi}=e^{\phi_{0}} \frac{1+q^{2} / n^{2}}{\left(1+\left[q^{2}+e^{2 \phi_{0}}\left(p-\chi_{0} q\right)^{2}\right] / n^{2}\right)^{1 / 2}},
$$

and the string-frame metric, in terms of this $g_{\mathrm{eff}}$, is

$$
\begin{aligned}
d s^{2}=\alpha^{\prime}[ & \frac{U^{2}}{\sqrt{4 \pi n g_{\mathrm{eff}}}} \frac{n^{2}+q^{2}}{n^{2}+q^{2}+e^{2 \phi_{0}}\left(p-\chi_{0} q\right)^{2}}\left(-\left(d x^{0}\right)^{2}+\left(d x^{1}\right)^{2}\right) \\
& +\frac{U^{2}}{\sqrt{4 \pi n g_{\mathrm{eff}}}}\left(1+q^{2} / n^{2}\right)\left(\left(d x^{2}\right)^{2}+\left(d x^{3}\right)^{2}\right) \\
& \left.+\sqrt{4 \pi n g_{\mathrm{eff}}}\left(\frac{d U^{2}}{U^{2}}+d \Omega_{5}^{2}\right)\right] .
\end{aligned}
$$

We are looking for an equivalent simple D3-brane description for this ((F, D1), D3) in the decoupling limit. We can achieve this minimally by rescaling $x^{0}$ and $x^{1}$ such that we end up with

$$
\begin{gathered}
d s^{2}=\alpha^{\prime}\left[\frac{U^{2}}{\sqrt{4 \pi n g_{\mathrm{eff}}}}\left(1+q^{2} / n^{2}\right)\left(-\left(d x^{0}\right)^{2}+\left(d x^{1}\right)^{2}+\left(d x^{2}\right)^{2}+\left(d x^{3}\right)^{2}\right)\right. \\
\left.+\sqrt{4 \pi n g_{\mathrm{eff}}}\left(\frac{d U^{2}}{U^{2}}+d \Omega_{5}^{2}\right)\right] .
\end{gathered}
$$

This metric has the isometries of $A d S_{5} \times S^{5}$ but not in the standard form because of the factor $\left(1+q^{2} / n^{2}\right)$. The metric in (4.4) therefore describes the near-horizon geometry of a simple D3 brane with 5 -form flux $n$ but with the string coupling given by $g_{\text {eff }}$ rather than the usual string coupling $g=e^{\phi_{0}}$. The extra factor $\left(1+q^{2} / n^{2}\right)$ in the above metric indicates that unlike the F-strings, the presence of D-strings in D3 brane worldvolume changes the entire D3 brane worldvolume metric. This factor does not play an important role in the near-horizon metric Eq. (4.4) but it does have influence on the asymptotic metric of the equivalent simple D3 brane as we will explain below. In the decoupling limit, D-brane 
picture implies that this equivalent simple D3 brane is also described by the $\mathcal{N}=4 U(n)$ SYM theory in $1+3$ dimensions with the gauge coupling $g_{\mathrm{YM}}^{2}=2 \pi g_{\mathrm{eff}}$ and the theta angle $\theta=4 \pi \chi_{\text {eff }}$ with $\chi_{\text {eff }}$ evaluated at the horizon as

$$
\chi_{\mathrm{eff}}=\left(p q+n^{2} \chi_{0}\right) /\left(n^{2}+q^{2}\right) .
$$

Thus in the same spirit of Maldacena, we should have an $A d S_{5} / C F T_{4}$ correspondence here but with the string coupling $g_{\text {eff }}$ and the axion $\chi_{\text {eff }}$.

This equivalent simple D3 brane is taken as the "effective" vacuum configuration for this correspondence. In analogy with the study of the decoupling limit of the (F, D3) bound state in [1], we may anticipate that this effective vacuum corresponds to ((F, D1), D3) bound state. In order to provide some evidence for this we notice that once the above coordinate rescalings are done, the information about the $(\mathrm{F}, \mathrm{D} 1)$ bound states in the $((\mathrm{F}, \mathrm{D} 1), \mathrm{D} 3)$ disappears in the resulting metric. However, unlike the case for (F, D3) discussed in [1], we have the extra factor $\left(1+q^{2} / n^{2}\right)$ appearing in the metric and therefore we should be careful in identifying $g_{\text {eff }}$ as the string coupling in the equivalent D3 brane. We discuss in the following how to make this identification in an unambiguous manner.

Note that in order to find a stable BPS configuration of D3 branes (in general $p$-branes) from the corresponding supergravity, we usually insist either the Einstein frame or the string frame metric to be asymptotically Minkowski. There actually exists a general choice for the asymptotic metric which includes the above two choices. Specifically, we can re-obtain the $p$-brane solutions in 12 by insisting the Einstein metric $g_{M N}$ to go to $e^{V_{0}} \eta_{M N}$ asymptotically with $V_{0}$ an arbitrary but given constant and $\eta_{M N}$ the flat Minkowski metric. For example, $V_{0}=0$ corresponds to choosing the Einstein metric to be asymptotically Minkowski while $V_{0}=-\phi_{0} / 2$ corresponds to choosing the string metric to be asymptotically Minkowski with $\phi_{0}$ the asymptotic value of the dilaton. Let us specialize for the D3 brane in type IIB theory. With this choice of asymptotic Einstein metric, we can repeat the calculation in [13] to have the following Einstein metric

$$
d s_{E}^{2}=e^{V_{0}}\left[H^{-1 / 2}\left(-\left(d x^{0}\right)^{2}+\cdots+\left(d x^{3}\right)^{2}\right)+H^{1 / 2} d y^{i} d y^{i}\right]
$$


where the Harmonic function $H$ is now

$$
H=1+\frac{4 \pi \alpha^{\prime 2} n}{r^{4}} e^{-2 V_{0}}
$$

and the integer $n$ is the 5 -form flux. If we denote the constant dilaton for this solution as $c_{0}$, then the string frame metric is

$$
d s^{2}=e^{c_{0} / 2+V_{0}}\left[H^{-1 / 2}\left(-\left(d x^{0}\right)^{2}+\cdots+\left(d x^{3}\right)^{2}\right)+H^{1 / 2} d y^{i} d y^{i}\right]
$$

As usual, in obtaining this metric we have set the brane $\sigma$-model coordinates $\sigma^{\mu}=x^{\mu}$ for $\mu=0,1,2,3$.

Now let us examine the near-horizon behavior of the above metric in the decoupling limit. We have $\left(U=\frac{r}{\alpha^{\prime}}=\right.$ fixed as $\left.\alpha^{\prime} \rightarrow 0\right)$

$$
d s^{2}=\alpha^{\prime}\left[\frac{U^{2}}{\sqrt{4 \pi n g_{s}}} g_{s} e^{2 V_{0}}\left(-\left(d x^{0}\right)^{2}+\cdots+\left(d x^{3}\right)^{2}\right)+\sqrt{4 \pi n g_{s}}\left(\frac{d U^{2}}{U^{2}}+d \Omega_{5}^{2}\right)\right]
$$

where $g_{s}=e^{c_{0}}$ is the present string coupling. We note from (4.9) that: 1) The coefficient in front of $d U^{2} / U^{2}$ or $d \Omega_{5}^{2}$ is always given by $\sqrt{4 \pi n g_{s}}$, independent of the constant $V_{0}$. This provides us a way to read off the string coupling unambiguously from a near-horizon geometry of D3 brane. 2) Also, unless $V_{0}=-c_{0} / 2$ (i.e., the string metric is asymptotically Minkowski), the near-horizon metric of a D3 brane is in general not in the standard form of $A d S_{5} \times S^{5}$. Now by comparing Eq. (4.4) with (4.9), we can see that the $g_{\text {eff }}$ is indeed the string coupling for the equivalent simple D3 brane and the asymptotic string metric for the equivalent D3 brane is not Minkowski but

$$
e^{V_{0}} \eta_{M N}=g_{\mathrm{eff}}^{-1 / 2}\left(1+q^{2} / n^{2}\right)^{1 / 2} \eta_{M N}
$$

We also note from Eqs.(4.4) and (4.9) that the differences between the equivalent D3 branes and the usual D3 branes (with string coupling $g=e^{\phi_{0}}$ and $V_{0}=-\phi_{0} / 2$ ) are in the string coupling constant and the asymptotic form of the metric. So the effects of these (F, D1) bound states must be encoded in the effective string coupling $g_{\text {eff }}$ given in Eq. (4.2) and the factor $1+q^{2} / n^{2}$. First let us examine the tension for the equivalent D3 brane. Since we 
have already determined its string coupling as $g_{\text {eff }}$, so the tension for this equivalent simple D3 brane is $n T_{0}^{3} / g_{\text {eff }}$. Now if we use the explicit expression for the $g_{\text {eff }}$, we find

$$
\frac{n T_{0}^{3}}{g_{\text {eff }}}=\frac{T_{0}^{3}}{g} \sqrt{n^{2}+q^{2}+g^{2}\left(p-\chi_{0} q\right)^{2}}\left(1+q^{2} / n^{2}\right)^{-1} .
$$

Comparing it with Eq. (3.3), we find that they differ by a factor $\left(1+q^{2} / n^{2}\right)^{-1}$. Recall that in obtaining (3.3), we calculated the tension against asymptotic D3 worldvolume whose metric is asymptotically Minkowski. In other words, the worldvolme is calculated according to $A_{4}=\int d^{4} \sigma$. However, the tension in Eq. (4.11) for the equivalent D3 brane is calculated with respect to its own asymptotic string metric which is in general not the same as the string metric for the original ((F, D1), D3) configuration. In the present case, the asymptotic string metric for the equivalent D3 brane is $g_{\text {eff }}^{1 / 2} e^{V_{0}} \eta_{M N}$ with $e^{V_{0}}$ given by Eq. (4.10). For $M, N=0,1,2,3$, it gives the worldvolume asymptotic metric. Therefore, the tension in Eq. (4.11) is calculated against the worldvolume $A_{4}^{\prime}=g_{\mathrm{eff}} e^{2 V_{0}} \int d^{4} \sigma$. In order to make a proper comparison with the tension (3.3), the present tension should be measured with respect to the same worldvolume, i.e., $A_{4}$ rather than $A_{4}^{\prime}$. In other words, we expect that

$$
\frac{n T_{0}^{3}}{g_{\text {eff }}} \frac{A_{4}^{\prime}}{A_{4}}
$$

should be the same as the tension of ((F, D1), D3) given by Eq. (3.3). This is indeed true since $A_{4}^{\prime} / A_{4}=\left(1+q^{2} / n^{2}\right)$. This indicates that the vacuum configuration for the $A d S_{5} / C F T_{4}$ correspondence in this case is the ((F, D1), D3) bound state.

On the SYM side, the $A d S_{5} / C F T_{4}$ correspondence suggested above implies that the electric and magnetic flux lines should disappear, too. As claimed, the gauge coupling and the theta angle should now be given as $g_{\mathrm{YM}}^{2}=2 \pi g_{\mathrm{eff}}$ and $\theta=4 \pi \chi_{\mathrm{eff}}$, respectively. Showing these two relations from the SYM side independently is certainly a non-trivial check of this correspondence. We will show below that these two relations are indeed true from the SYM side.

We know from D-brane picture that the dynamics of a single D3 brane (or $n$ coincident D3 branes) is described in general, in the low energy limit, by the abelian (or non-abelian 
$U(n))$ Born-Infeld action of $\mathcal{N}=4$ SYM coupled with background fields. In the decoupling limit, these background fields are decoupled and therefore the D3 brane (or $n$ coincident D3 branes) is described purely by the gauge fields with flat Minkowski background. Since the non-abelian version of the Born-Infeld action for $n$ coincident D3 branes is not known yet we limit ourselves here to the abelian Born-Infeld action of a single D3 brane. This turns out to be sufficient for confirming the abovementioned relations.

Our worldvolume study in [3] and the spacetime study in [1] show that the F-strings and D-strings in $((\mathrm{F}, \mathrm{D} 1), \mathrm{D} 3)$ correspond to the quantized constant electric field $\left(2 \pi \alpha^{\prime}\right) E=$ $g\left(p-q \chi_{0}\right)$ and the quantized constant magnatic field $\left(2 \pi \alpha^{\prime}\right) B=q$, respectively, in the linear approximation' For a single D3 brane (i.e., $n=1$ ), while the linear approximation can be justified for the electric field $E$ by insisting a small string coupling $g$, this approximation is not valid, strictly speaking, for the magnetic field $B$ with a non-vanishing integer $q$. Even so, we obtain the correct tension for the bound state ((F, D1), D3). The success in obtaining the tension for $((\mathrm{F}, \mathrm{D} 1), \mathrm{D} 3)$ even for $n \neq 1$ seems to indicate that we may take the $n$ coincident D3 branes effectively as a single D3 brane with a tension $n T_{0}^{3}$ and the constant electric field as $\left(2 \pi \alpha^{\prime}\right) E=g\left(p-q \chi_{0}\right) / n$ and the constant magnetic field as $\left(2 \pi \alpha^{\prime}\right) B=q / n$ at least for these two constant background fields. If we assume this, the linear approximation can always be made by insisting, for example, $n>>q, n>>p$, with small $g$. We will take these effective $E$ and $B$ as our constant background fields where $n=1$ is just a special case.

Let us recall that in [3], we picked a special direction, i.e, the $x^{1}$-direction, as the direction for the quantized constant electric and magnetic fields. By doing so, we break the Lorentz $S O(1,3)$ to $S O(1,1) \times S O(2)$. In order to find the corresponding description of the bulk equivalent simple D3 brane, we now need to make a consistent ansatz for the background

\footnotetext{
${ }^{3} \mathrm{~A}$ possible form was suggested by Tseylin 114.

${ }^{4}$ Even though these electric and magnetic fields are good only in the linear approximation, the tension obtained in [3] is actually exact. This is the property of a BPS state.
} 
gauge fields which respects the $S O(1,3)$ symmetry as well as the translational symmetry. This can be achieved in the following two ways. We can take a stochastic averaging of a constant homogeneous background as in [7]. Or we can follow [15] to take the constant homogeneous background as the ground-state expectation value. We here follow the latter approach and have

$$
\begin{aligned}
& <\mathcal{F}_{\mu \nu}>=0 \\
& \left(2 \pi \alpha^{\prime}\right)^{2}<\mathcal{F}_{\mu \nu} \mathcal{F}^{\lambda \sigma}>=\frac{q^{2}-g^{2}\left(p-q \chi_{0}\right)^{2}}{6 n^{2}} \delta_{\mu \nu}^{\lambda \sigma}-\frac{g q\left(p-q \chi_{0}\right)}{3 n^{2}} \epsilon_{\mu \nu}{ }^{\lambda \sigma},
\end{aligned}
$$

where $\left\langle\cdots>\right.$ denotes the vacuum expectation value of field operators, $\delta_{\mu \nu}^{\lambda \sigma}=\delta_{\mu}^{\lambda} \delta_{\nu}^{\sigma}-\delta_{\nu}^{\lambda} \delta_{\mu}^{\sigma}$ and $\epsilon^{\mu \nu \lambda \sigma}$ is the totally antisymetric tensor with $\epsilon^{0123}=1(\mu, \nu, \lambda, \sigma=0,1,2,3)$. In the above, we first have $\left\langle\mathcal{F}^{2}\right\rangle=2 a$ and $\langle\mathcal{F} \tilde{\mathcal{F}}\rangle=4 b$ with $a$ and $b$ the corresponding constant VEV's. Then we determine the right side of the above second equation by insisting $a=\left(B^{2}-E^{2}\right)$ and $b=E B$ with $E$ and $B$ the background fields given before. Also, here $\tilde{\mathcal{F}}_{\mu \nu}=(1 / 2) \epsilon_{\mu \nu}{ }^{\lambda \sigma} \mathcal{F}_{\lambda \sigma}$.

Since our purpose is to find the effective gauge coupling and the effective theta angle for the fluctuations of gauge fields with respect to the above background, so we need to consider only the gauge fields in the BI action with the Lagrangian

$$
\mathcal{L}=-\frac{T_{3}}{g} \sqrt{-\operatorname{det}\left(\eta_{\mu \nu}+\left(2 \pi \alpha^{\prime}\right) \mathcal{F}_{\mu \nu}\right)}+\frac{\chi_{0} T_{3}}{8}\left(2 \pi \alpha^{\prime}\right)^{2} \epsilon^{\mu \nu \lambda \sigma} \mathcal{F}_{\mu \nu} \mathcal{F}_{\lambda \sigma}
$$

where $g=e^{\phi_{0}}$ and $\chi_{0}$ are the asymptotic string coupling and the asymptotic value of the RR scalar, respectively, and $T_{3}=n T_{0}^{3}$.

Now we write $\mathcal{F}_{\mu \nu}=f_{\mu \nu}+F_{\mu \nu}$ with $f_{\mu \nu}$, the fluctuating field and $F_{\mu \nu}$, the background, i.e., $<f_{\mu \nu}>=0$ and $<f_{\mu \nu} f^{\lambda \sigma}>=0$ and expand the above Lagrangian, with respect to the background, to quadratic order in $f_{\mu \nu}$. We end up with a quadratic Lagrangian of $f_{\mu \nu}$ as

$$
\mathcal{L}_{f}=\frac{1}{8}<\frac{\partial^{2} \mathcal{L}}{\partial \mathcal{F}_{\mu \nu} \partial \mathcal{F}^{\lambda \sigma}}>f_{\mu \nu} f^{\lambda \sigma}
$$

Where,

\footnotetext{
${ }^{5}$ Non-vanishing $<\mathcal{F} \tilde{\mathcal{F}}>$ implies the spontaneous breaking of $\mathrm{P}$ and $\mathrm{CP}$ invariances.
} 


$$
\begin{aligned}
<\frac{\partial^{2} \mathcal{L}}{\partial \mathcal{F}_{\mu \nu} \partial \mathcal{F} \lambda \sigma}>= & \frac{\left(2 \pi \alpha^{\prime}\right)^{2} T_{3}}{g}\left[-\frac{1}{4}\left(2 \pi \alpha^{\prime}\right)^{2}<\mathcal{F} \tilde{\mathcal{F}}>\epsilon_{\lambda \sigma}^{\mu \nu}+\left(1-\frac{1}{4}\left(2 \pi \alpha^{\prime}\right)^{2}<\mathcal{F}^{2}>\right) \delta_{\lambda \sigma}^{\mu \nu}\right] \\
& +T_{3}\left(2 \pi \alpha^{\prime}\right)^{2} \epsilon^{\mu \nu}{ }_{\lambda \sigma}, \\
=n & {\left[-\frac{1}{2 \pi g}\left(1-\frac{q^{2}}{2 n^{2}}+\frac{g^{2}\left(p-q \chi_{0}\right)^{2}}{2 n^{2}}\right) \delta_{\lambda \sigma}^{\mu \nu}\right.} \\
& \left.+\frac{1}{2 \pi}\left(\chi_{0}+\frac{q\left(p-q \chi_{0}\right)}{n^{2}}\right) \epsilon_{\lambda \sigma}^{\mu \nu}\right] .
\end{aligned}
$$

Note that since the background fields in [3] were obtained upto the quadratic order in gauge fields, we have evaluated $<\frac{\partial^{2} \mathcal{L}}{\partial \mathcal{F}_{\mu \nu} \partial^{\mathcal{F}^{\lambda \sigma}}}>$ in the first expression of Eq.(4.16) upto that order. Also note that we have used $\left.\left\langle\mathcal{F}^{2}\right\rangle+<\tilde{\mathcal{F}}^{2}\right\rangle=0$ and

$$
\operatorname{det}\left(\eta_{\mu \nu}+\left(2 \pi \alpha^{\prime}\right) \mathcal{F}_{\mu \nu}\right)=-\left[1+\frac{1}{2}\left(2 \pi \alpha^{\prime}\right)^{2} \mathcal{F}^{2}-\frac{1}{16}\left(2 \pi \alpha^{\prime}\right)^{4}(\mathcal{F} \tilde{\mathcal{F}})^{2}\right]
$$

It should be pointed out that in obtaining the second expression of Eq.(4.16), we have used Eq. (4.13) and $T_{3}=n T_{0}^{3}=n /\left((2 \pi)^{3} \alpha^{\prime 2}\right)$. We therefore find the effective Lagrangian to this order to be of the form,

$$
\mathcal{L}_{f} / n=-\frac{1}{8 \pi g}\left(1-\frac{q^{2}}{2 n^{2}}+\frac{g^{2}\left(p-q \chi_{0}\right)^{2}}{2 n^{2}}\right) f_{\mu \nu} f^{\mu \nu}+\frac{1}{16 \pi}\left(\chi_{0}+\frac{q\left(p-q \chi_{0}\right)}{n^{2}}\right) \epsilon^{\mu \nu \lambda \sigma} f_{\mu \nu} f_{\lambda \sigma}
$$

where the overall integer $n$ indicates that we have $n$ copies of the $U(1)$ Lagrangian. We can read, from the above action, the gauge coupling and the theta angle, as

$$
\begin{aligned}
\frac{1}{g_{\mathrm{YM}}^{2}} & =\frac{1}{2 \pi g}\left(1-\frac{q^{2}}{2 n^{2}}+\frac{g^{2}\left(p-q \chi_{0}\right)^{2}}{2 n^{2}}\right), \\
\theta & =4 \pi\left(\chi_{0}+\frac{q\left(p-q \chi_{0}\right)}{n^{2}}\right)
\end{aligned}
$$

which, in the linear approximation, agree completely with the predictions of the $A d S_{5} \times S^{5}$ correspondence, i.e., $g_{\mathrm{YM}}^{2}=2 \pi g_{\text {eff }}$ and $\theta=4 \pi \chi_{\text {eff }}$, respectively. We can examine this simply

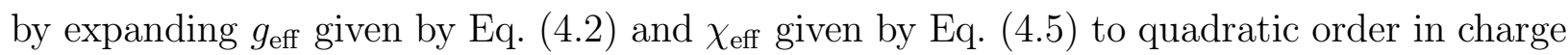
$p$ and $q$ as:

$$
\frac{1}{g_{\mathrm{eff}}}=\left[\frac{1}{g}-\frac{1}{2 g}\left(\frac{q}{n}\right)^{2}+\frac{1}{2 g}\left(\frac{g\left(p-\chi_{0} q\right)}{n}\right)^{2}\right]
$$


and

$$
\chi_{\mathrm{eff}}=\chi_{0}+\frac{q\left(p-q \chi_{0}\right)}{n^{2}}
$$

So we confirm the predictions of the $A d S_{5} \times S^{5}$ correspondence between the SYM coupling and the effective string coupling and between the theta angle and the value of the RR scalar evaluated at the horizon. In analogy with the bulk picture of the F-strings and D-strings in the decoupling limit, the effects of the electric and magnetic background fields are absorbed into the gauge coupling constant $g_{\mathrm{YM}}^{2}=2 \pi g_{\mathrm{eff}}$ and the theta angle $\theta=4 \pi \chi_{\text {eff }}$ and they do not appear in the resulting SYM theory. Therefore, we expect that the superconformal symmetry is then restored. Thus we have a consistent picture both in the bulk and on the brane.

Given the $A d S_{5} / \mathrm{CFT}_{4}$ correspondence with respect to the equivalent simple D3 brane, we should have an $A d S_{5} / C F T_{4}$ correspondence with respect to the ((F, D1), D3) configuration with the string coupling $g_{\mathrm{eff}}$ and the gauge coupling $g_{\mathrm{YM}}^{2}=2 \pi g_{\mathrm{eff}}$. This in turn indicates that Maldacena's $A d S_{5} / C F T_{4}$ correspondence holds true even for this non-trivial D3 brane configuration but now with the string coupling $g_{\text {eff }}$ rather than the usual string coupling $g$.

As in the case of (F, D3) bound state, the effective string coupling in Eq.(3.6) is again quantized but now in terms of integers $p, q, n$. From Eq. (4.2) we find that the F-strings in ((F, D1), D3) tend to decrease the coupling while the D-strings tend to increase it. Therefore, we may control the string coupling in the near-horizon region by adding or subtracting Fstrings (or D-strings).

\section{T-DUAL DAUGHTERS OF ((F, D1), D3)}

Once we have the space-time configuration for the non-threshold ((F, D1), D3) bound state as given in section 2, its descendants can be obtained from this by T-dualities, as described in detail in [1]. T-dualities can be applied either along the transverse directions

of the D3 brane in $((\mathrm{F}, \mathrm{D} 1)$, D3) or along the longitudinal directions of the D3 brane 
which includes the direction of the (F, D1)-strings. Even though these configurations can, in principle, be obtained directly from the equations of motion of type IIA or type IIB supergravity theory, it would be very difficult to obtain them in practice if we do not have these symmetries in hand. There is no doubt that these configurations will play an important role in the formulation of the unique M- or U-theory.

In this section, we will first discuss all possible non-threshold bound states which can be obtained from ((F, D1), D3) by T-dualities[? following the table given below:

\begin{tabular}{|c|c|c|}
\hline & Parallel & Transverse \\
\hline $\mathrm{Dp}$ & $\mathrm{D}(\mathrm{p}-1)$ & $\mathrm{D}(\mathrm{p}+1)$ \\
$\mathrm{F}$ & $\mathrm{W}$ & $\mathrm{F}$ \\
$\mathrm{W}$ & $\mathrm{F}$ & $\mathrm{W}$ \\
$\mathrm{NS} 5$ & $\mathrm{NS} 5$ & $\mathrm{KK}$ \\
$\mathrm{KK}$ & $\mathrm{KK}$ & $\mathrm{NS} 5$ \\
\hline
\end{tabular}

In this table W, F, NS5 and KK denote waves, fundamental strings, NS fivebranes, and KK monopoles, respectively, and they are associated with NSNS fields. Dp $(-1 \leq p \leq 8)$ are the so-called D-branes and are associated with the RR fields.

Then we will present explicit Einstein-frame metric and dilaton for each of the nonthreshold ((F, Dp), D $(\mathrm{p}+2))$ bound states for $2 \leq p \leq 5$ where the well-known $(\mathrm{Dp}, \mathrm{D}(\mathrm{p}$ $+2)$ ) bound states are just special cases.

Let us begin with the T-dualities along the transverse directions of the D3 brane. We denote the coordinates along the D3 brane as $x^{0}, x^{1}, x^{2}, x^{3}$, and 4, 5, 6, 7, 8, 9 as its transverse directions. We also denote $\left(\mathrm{T}_{i}: \mapsto\right)$ as the T-duality along the $i$-th direction. According to the above table, we have

$$
\begin{aligned}
& ((\mathrm{F}, \mathrm{D} 1), \mathrm{D} 3)\left(\mathrm{T}_{4}: \mapsto\right)((\mathrm{F}, \mathrm{D} 2), \mathrm{D} 4)\left(\mathrm{T}_{5}: \mapsto\right)((\mathrm{F}, \mathrm{D} 3), \mathrm{D} 5)\left(\mathrm{T}_{6}: \mapsto\right)((\mathrm{F}, \mathrm{D} 4), \mathrm{D} 6) \\
& \left(\mathrm{T}_{7}: \mapsto\right)((\mathrm{F}, \mathrm{D} 5), \mathrm{D} 7)\left(\mathrm{T}_{8}: \mapsto\right)((\mathrm{F}, \mathrm{D} 6), \mathrm{D} 8) .
\end{aligned}
$$

\footnotetext{
${ }^{6}$ We do not consider any Dp brane resulting from D9 brane.
} 
We can T-dualize each of the above $((\mathrm{F}, \mathrm{Dp}), \mathrm{D}(\mathrm{p}+2))$ for $1 \leq p \leq 6$ along the longitudinal directions of the original D3 brane, i.e., 1, 2, 3 directions. We end up with different bound states depending on whether we T-dualize along the ' 1 ' direction first or not. We demonstrate this on $((F, D 1), D 3)$ as examples. The other cases just follow. If we T-dualize ((F, D1), D3) along the '1', i.e., the direction of (F, D1)-strings, we end up with ((W, D0), D2). If we T-dualize along the '3', we have ((F, D2), D2). We can now T-dualize on ((F, D2), D2) along either '2' or ' 1 '. In the former case, we have ((F, D3), D1) while for the latter we have ((W, D1), D1). We can further T-dualize ((F, D3), D1) along the '1', we end up with ((W, D2), D0).

In general, we have $((\mathrm{W}, \mathrm{D}(\mathrm{p}-1)), \mathrm{D}(\mathrm{p}+1))$ if we T-dualize along ' 1 ' direction first on the $((\mathrm{F}, \mathrm{Dp}), \mathrm{D}(\mathrm{p}+2))$ for $1 \leq p \leq 6$. If we T-dualize first along ' 3 ' instead, we have $((\mathrm{F}, \mathrm{D}(\mathrm{p}+1)), \mathrm{D}(\mathrm{p}+1))$. If we T-dualize on $((\mathrm{F}, \mathrm{D}(\mathrm{p}+1)), \mathrm{D}(\mathrm{p}+1))$ along either ' 1 ' or ' 2 ', we end up with $((\mathrm{W}, \mathrm{Dp}), \mathrm{Dp})$ or $((\mathrm{F}, \mathrm{D}(\mathrm{p}+2))$, Dp), respectively. Further we can T-dualize on $((F, D(p+2))$, Dp) along ' 1 ', we end up with $((\mathrm{W}, \mathrm{D}(\mathrm{p}+1)), \mathrm{D}(\mathrm{p}-1))$. We summarize all the bound states obtained this way in the following table where the second column indicates the common directions shared by the two D branes in the corresponding bound state:

\begin{tabular}{|c|c|}
\hline Bound States & No. Common Dir. \\
\hline$((\mathrm{W}, \mathrm{D}(\mathrm{p}-1)), \mathrm{D}(\mathrm{p}+1))$ & $p-1$ \\
$((\mathrm{~W}, \mathrm{D}(\mathrm{p}+1)), \mathrm{D}(\mathrm{p}-1))$ & $p-1$ \\
$((\mathrm{~W}, \mathrm{D} \mathrm{p}), \mathrm{D} \mathrm{p})$ & $p-1$ \\
$((\mathrm{~F}, \mathrm{D}(\mathrm{p}+1)), \mathrm{D}(\mathrm{p}+1))$ & $p$ \\
$((\mathrm{~F}, \mathrm{D}(\mathrm{p}+2)), \mathrm{D} p)$ & $p$ \\
$((\mathrm{~F}, \mathrm{D} p), \mathrm{D}(\mathrm{p}+2))$ & $p$ \\
\hline
\end{tabular}

These are all possible non-threshold bound states which can be obtained from ((F, D1), D3) by T-dualities. Among these bound states, the well-known $(\mathrm{Dp}, \mathrm{D}(\mathrm{p}+2))$ bound states for $0 \leq p \leq 6$ [16] appear as special cases when the charges associated with either F-strings 
or $\mathrm{W}$-waves are set to zero. If we set the charge associated with the $\mathrm{W}$-waves in ((W, Dp), $\mathrm{Dp})$ or the F-strings in $((\mathrm{F}, \mathrm{D}(\mathrm{p}+1)), \mathrm{D}(\mathrm{p}+1))$ for $1 \leq p \leq 6$ to zero, we end up with non-threshold (Dp, Dp) bound states $1 \leq p \leq 7$ which appear to be new. As mentioned above, the two Dp's share only $(p-1)$ common directions. For those in the type IIB theory, we can apply SL(2,Z) to obtain new bound states. For examples, from ((F, D3), D3), we can have $(((\mathrm{F}, \mathrm{D} 1), \mathrm{D} 3), \mathrm{D} 3)$. One can also perform the consistency checks of the $A d S_{5} / C F T_{4}$ correspondence for these two bound states.

If we T-dualize (((F, D1), D3), D3) along the common direction of the two D3's only, we have $(((F, D 2), D 2), D 2)$. If we now set the charge associated with the F-strings to zero, we end up with a non-threshold bound state (D2, D2, D2). The three D2's share only one common direction. We can obtain (D1, D1, D1) by T-dualizing along the common direction of the three D2's or we can obtain(Dp, Dp, Dp) for $3 \leq p \leq 7$ by T-dualizing along directions transverse to (D2, D2, D2). The three Dp's in (Dp, Dp, Dp) for $1 \leq p \leq 7$ share $p-1$ common directions. We can also obtain (((F, D3), D3), D3) by T-dualizing along one of directions transverse to (((F,D2), D2), D2). Then we apply SL(2,Z) to (((F, D3), D3), D3), we can obtain $((((F, D 1), D 3), D 3), D 3)$. If we T-dualize this state along the common direction of the three D3's only, we obtain $((((F, D 2), D 2), D 2), D 2)$. By setting the charge associated the F-strings to zero, we end up with the non-threshold bound state (D2, D2, D2, D2). We repeat what we have done for (D2, D2, D2). We can have non-threshold bound states (Dp, Dp, Dp, Dp) for $1 \leq p \leq 6$ where the four Dp's share $p-1$ common directions. We can continue this process to have bound states (Dp, Dp, Dp, Dp, Dp) for $1 \leq p \leq 5$ and so on. Finally, in this way we can have a seemingly unique bound state (D1, D1, D1, D1, D1, D1, D1, D1, D1) where the nine D1's share no common direction.

Also, from ((F, D5), D5), we can have (( F, D1), (NS5, D5)), (NS5, D5)) by S-duality of type IIB theory. As we will demonstrate in a bit simpler case of this, i.e., ((F, D1), (NS5, D5)), in [17] that the charges for the D5- and NS5-branes are related to the charges for the F-strings and D-strings. This may suggest that the formation of the $\operatorname{SL}(2, \mathrm{Z})$ fivebrane bound states [8, 18, 19] be closely related to that of $(\mathrm{F}, \mathrm{D} 1)$ bound states or $(m, n)$-strings. 
From ((F, D3), D5), we can have (((F, D1), D3), (NS5, D5)) and so on.

Once we have these new bound states, we can again apply T-dualities to obtain other new bound states, then S-dualities on those in type IIB. We can continue this process to obtain all possible non-threshold bound states by S- and T-dualities simply from the original (F, D1)-strings. All these non-threshold bound states preserve one half of the spacetime supersymmetries. Even though we are, at this point, unable to count how many of these bound states are there, we believe that the total number of these bound states is finite and may be related to the number of generators of the largest finite U-duality group [11] in type II theory, i.e., $E_{8(+8)}$. After all, these bound states are obtained by repeated applications of the S- and T-dualities. We speculate here that these bound states will form the multiplets of the $E_{8(+8)}$ U-duality symmetry in the yet unknown M- or U-theory. We wish to come back to provide more evidence for this elsewhere.

In closing this section we provide, as examples, the explicit Einstein-frame metric and the dilaton for each of the $((\mathrm{F}, \mathrm{D}(\mathrm{p}-2))$, $\mathrm{Dp})$ bound states for $3 \leq p \leq 7$. The metric is

$$
\begin{aligned}
d s^{2}= & e^{-\phi_{0} / 2}\left(H H^{\prime}\right)^{1 / 4} H^{\prime \prime(p-3) / 8}\left[H^{-1}\left(-\left(d x^{0}\right)^{2}+\left(d x^{1}\right)^{2}\right)+H^{\prime-1}\left(\left(d x^{2}\right)^{2}+\left(d x^{3}\right)^{2}\right)\right. \\
& \left.+H^{\prime \prime-1}\left(\left(d x^{4}\right)^{2}+\cdots+\left(d x^{p}\right)^{2}\right)+d y^{i} d y^{i}\right]
\end{aligned}
$$

with $i=1, \cdots, 9-p$; and the dilaton is

$$
e^{\phi}=e^{\phi_{0}}\left(H^{\prime} H\right)^{-1 / 2} H^{\prime \prime(7-p) / 4} \text {. }
$$

In the above, we have chosen the string-frame metric to be asymptotically Minkowski. The Harmonic function $H$ is

$$
H= \begin{cases}1+\frac{Q_{p}}{r^{7-p}}, & 3 \leq p \leq 6, \\ 1-Q_{7} \ln r, & p=7,\end{cases}
$$

with $Q_{p}=\Delta_{(p, q, n)}^{1 / 2} e^{3 \phi_{0} / 2} \sqrt{2} \kappa_{0} /\left[(7-p) \Omega_{8-p}\right]$ and the Harmonic functions $H^{\prime}$ and $H^{\prime \prime}$ are

$$
\begin{aligned}
H^{\prime} & =\left[\left(n^{2} H+q^{2}\right) e^{-\phi_{0}}+\left(p-\chi_{0} q\right)^{2} e^{\phi_{0}}\right] \Delta_{(p, q, n)}^{-1}, \\
H^{\prime \prime} & =\frac{q^{2} H e^{-\phi_{0}}+\left(p-\chi_{0} q\right)^{2} H^{\prime} e^{\phi_{0}}}{q^{2} e^{-\phi_{0}}+\left(p-\chi_{0} q\right)^{2} e^{\phi_{0}}} .
\end{aligned}
$$


Other fields can be obtained from those given in section 3 for the ((F, D1), D3) configuration, following the prescription given in [1]. In the above, the $\Delta$-factor continues to be given by Eq. (3.1) but now the integer $p$ represents the quantized charge per $(2 \pi)^{p-1} \alpha^{\prime(p-1) / 2}$ area over the $x^{2} \cdots x^{p}$-plane carried by the F-strings, the integer $q$ represents the quantized charge per $(2 \pi)^{2} \alpha^{\prime}$ area over the $x^{p-1} x^{p}$-plane carried by the $\mathrm{D}(\mathrm{p}-2)$ branes, and the integer $n$ is the

quantized $(p+2)$-form charge carried by the Dp brane. The tension for each of these bound states can be calculated by generalizing the mass per unit $p$-brane volume given in [20] in a similar fashion as we did in [1]. The result is

$$
T_{p}(p, q, n)=\frac{T_{0}^{p}}{g} \sqrt{n^{2}+q^{2}+g^{2}\left(p-\chi_{0} q\right)^{2}},
$$

where the subscript ' $p$ ' in $T_{p}$ and the superscript ' $p$ ' in $T_{0}^{p}$ should not be confused with the integral charge $p$ for the F-strings.

\section{CONCLUSION}

To conclude, we have constructed in this paper the explicit space-time configuration for the non-threshold ((F, D1), D3) bound state using the type IIB SL(2,Z) symmetry and the known (F, D3) configuration. We have shown that the ((F, D1), D3) bound state is identical to the bound state consisting of D3 branes carrying quantized constant electric and magnetic fields. The $(p, q)$-strings are identical to the corresponding electric and magnetic, or dyonic, flux lines. This also connects the type IIB SL(2,Z) symmetry in the bulk with the SL(2,Z) symmetry in the SYM theory on the brane. We have studied the decoupling limit of this bound state. We found that given the $A d S_{5} / C F T_{4}$ correspondence of Maldacena with respect to a simple D3 brane and the usual string coupling, we should have a similar correspondence with respect to this bound state but now with an effective string coupling. This, in turn, indicates that Maldacena's $A d S_{5} / C F T_{4}$ correspondence holds true even for a non-trivial D3 brane configuration with the corresponding effective string coupling rather than the usual string coupling. We also found that D-strings in ((F, D1), D3) bound state 
tend to increase the effective string coupling while the F-strings tend to decrease it. This property may be used to control the string coupling in the bulk and the SYM coupling on the brane in the near-horizon region.

We have given the list of all possible descendants of this ((F, D1), D3) bound states by T-dualities. Unless there is an obstacle preventing us to perform T-dualities and the type IIB $\mathrm{SL}(2, \mathrm{Z})$ on these bound states, we have predicted the existence of new non-threshold bound states (Dp, Dp) for $1 \leq p \leq 8$ where the two Dp's share only $(p-1)$ common dimensions, (Dp, Dp, Dp) for $1 \leq p \leq 7$ with the three Dp's sharing $p-1$ common directions, (Dp, Dp. Dp, Dp) for $1 \leq p \leq 6$ with the four Dp's sharing $p-1$ common directions and so on. This process stops at the seemingly unique bound state (D1, D1, D1, D1, D1, D1, D1, D1, D1) with the nine D1's sharing no common direction. Moreover, we have presented the explicit configurations for the $((\mathrm{F}, \mathrm{Dp}), \mathrm{D}(\mathrm{p}+2))$ bound states for $2 \leq p \leq 5$. Using the type IIB SL(2,Z) symmetries on those newly obtained bound states, we can construct further complicated bound states. Then by T-dualities, we can have even more bound states. We can probably exhaust all possible bound states by continuing this process. All these bound states are BPS ones, preserving one half of the spacetime supersymmetries. These bound states can, in principle, be obtained from the equations of motion without the need to use the S- and T-dualities. They are expected to appear in the uncompactified M- or U-theory. We have conjectured that the total number of these bound states are finite and these bound states will form multiplets of the U-duality group $E_{8(+8)}$ in the yet unknown M- or U-theory.

\section{ACKNOWLEDGMENTS}

JXL acknowledges the support of NSF Grant PHY-9722090. We thank Juan Maldacena for an e-mail correspondence. 


\section{REFERENCES}

[1] J. X. Lu and S. Roy, "Non-threshold (F, Dp) bound states", hep-th/9904129.

[2] J. Maldacena, Adv. Theor. Math. Phys. 2 (1998) 231.

[3] J. X. Lu and S. Roy, "(m,n)-string-like Dp-brane bound states", hep-th/9904112.

[4] T. Banks and M. Green, JHEP 9805 (1998) 002, hep-th/9804170.

[5] E. Witten, JHEP 9807 (1998) 006, hep-th/9805112.

[6] C. -S. Chu, P. -M. Ho and Y. -Y. Wu, Nucl. Phys. B541 (1999) 179, hep-th/9806103.

[7] H. Liu and A. A. Tseytlin, "D3-brane D instanton configuration and N=4 super YM theory in constant selfdual background", hep-th/9903091.

[8] J. Schwarz, Phys. Lett. B360 (1995) 13 (see hep-th/9508143 for the most recent revision of this paper).

[9] J. X. Lu and S. Roy, Nucl. Phys. B538 (1999) 149.

[10] E. Cremmer and B. Julia, Phys. Lett. B80 (1987) 48, Nucl. Phys. B156 (1979) 141, B. Julia, in Superspace and Supergravity, Eds. S. W. Hawking and M. Rocek (Cambridge University Press, Cambridge, 1981), J. Schwarz, Nucl. Phys. B226 (1983) 269.

[11] C. M. Hull and P. K. Townsend, Nucl. Phys. B294 (1995) 196.

[12] M. J. Duff, R. Khuri and J. X. Lu, Phys. Rept. 259 (1995) 213.

[13] M. J. Duff and J. X. Lu, Phys. Lett. B273 (1991) 409.

[14] A. A. Tseylin, "On non-abelian generalization of Born-Infeld action in string theory", hep-th/9701125.

[15] P. Minkowski, Nucl. Phys. B177 (1981) 203.

[16] J. Polchinski, String Theory, Vol.II (Cambridge University Press, 1998), M. Green and 
M. Gutperle, Nucl. Phys. B476 (1996) 484, G. Lifschytz, Nucl. Phys. B499 (1997) 283, M. B. Green, N. D. Lambert, G. Papadopoulos and P. K. Townsend, Phys. Lett. B384 (1996) 86; J. G. Russo and A. A. Tseytlin, Nucl. Phys. B490 (1997) 121; J. Breckenridge, G. Michaud and R. Myers, Phys. Rev. D55 (1997) 6438, H. Arfaei and M. M. Sheikh Jabbari, Nucl. Phys. B526 (1998) 278.

[17] J. X. Lu and S. Roy, "(F, D5) bound state, SL(2,Z) invariance and the descendant states in type IIB/IIA string theory", hep-th/9905056.

[18] E. Witten, Nucl. Phys. B460 (1996) 335, hep-th/9510135.

[19] J. X. Lu and S. Roy, Phys. Lett. B428 (1998) 289.

[20] J. X. Lu, Phys. Lett. B313 (1993) 29. 